\title{
Renal tubular acidosis in chronic liver disease
}

\author{
Peter L. Golding \\ M.B., B.S., M.R.C.P. \\ Royal Portsmouth Hospital, Portsmouth, Hants
}

\begin{abstract}
Summary
Renal tubular acidosis of the gradient or classic type, thought to be due to a disorder of the distal tubule, has been found to occur in $32 \%$ of 117 patients with chronic liver disease. Whilst the cause of this disorder is probably multifactorial, immunological mechanisms are considered to play a major role. The presence of this disorder might well be a cause, rather than the result of, the various electrolyte abnormalities seen in patients with chronic liver disease.
\end{abstract}

RENAL tubular acidosis (RTA) is a disorder of function in which the renal tubule is unable to acidify urine normally out of proportion to any reduction in the glomerular filtration rate. In a review by Morris in 1969 he reclassified and summarized the main features of two major types of tubular defect. In one there is an abnormality of the proximal tubular function in which there is a rate defect in the acidification process for the proximal tubule and more than $15 \%$ of the filtered bicarbonate load is excreted and the $\mathrm{THCO}_{3}$ - is at or near its maximum at normal plasma bicarbonate concentration. This type characteristically occurs as one of several proximal tubular dysfunctions but may appear as an isolated defect. This is referred to as bicarbonate wasting RTA or rate RTA. The other type of tubular defect is where the distal tubule is unable to maintain the steep lumen peritubular hydrogen ion gradient and this has been referred to as classic or gradient RTA. This latter condition is found in association with many other diseases and abnormal metabolic states, as shown in Table 1. Of particular interest in this table is that RTA is sometimes found in association with hypergammaglobulinaemic states and also in conditions where there is hyponatraemia or hypokalaemia. These disorders are commonly to be found in patients with chronic liver disease and have been suggested as aetiological agents in the sporadic cases of renal tubular acidosis reported in association with liver disease (Morris and Fudenberg, 1967; Read, Sherlock and Harrison, 1963; Seedat and Raine, 1965; Leeson and Fourman, 1967; Shear, Bonkowsky and Gabuzda, 1969). To determine the prevalence and nature of the tubular defect in patients with
TABle 1. Causes of Renal Tubular Acidosis (RTA)gradient RTA; distal tubule

Primary
Sporadic
Genetically transmitted
Genetically transmitted systemic diseases
Galactosaemia
Hereditary fructose intolerance
Ehlers-Danlos syndrome
Metabolic disorders
$\quad$ Hyperthyroidism with nephrocalcinosis
Primary hyperparathyroidism
Hypergammaglobulinaemic states
Medullary sponge kidney
Drug
Amphotericin, vitamin D-induced nephrocalcinosis
Pyelonephritis (?)
Renal transplantation
Electrolyte imbalance
Hyponatraemia
Massive diuretics
Hypokalaemia

chronic liver disease a systematic study was undertaken and the earlier results of this were reported when it was shown that the distal or gradient type of renal tubular acidosis occurred commonly in patients with primary biliary cirrhosis, active chronic hepatitis and cryptogenic cirrhosis (Golding and Mason, 1971). This report is an extension of the previous study.

\section{Patients and methods}

One hundred and seventeen patients attending Southampton General Hospital, The London Hos-윽 pital, St Bartholomew's Hospital and King's College $>$ Hospital, were studied for tubular defects. Distal tubular acidification defects can occur in two ways, $N$ either presenting as an overt acidosis in which thepatient has a metabolic acidosis but is unable to $N$ acidify the urine, or as an incomplete variety where $N$ the tubular defect can only be demonstrated when the patient is given an acid load. Overt renal tubulare acidosis was diagnosed in those patients who had a metabolic acidosis and whose urine pH was 6 or $\stackrel{5}{+}$ greater. Under these conditions it was considered unnecessary to give them a further acid loading stress. To detect the incomplete variety, the acid 
loading test of Wrong and Davies (1959) was used. The patient was given an ammonium chloride load and the urine was collected over $2 \mathrm{hr}$ periods for $8 \mathrm{hr}$. Each aliquot was analysed for urinary $\mathrm{pH}$, titratable acidity and total ammonia excretion. In a normal individual the urine $\mathrm{pH}$ falls to $<5.2$ during the test, the titratable acidity rises to $>24 \mu \mathrm{Eq} / \mathrm{min}$ and the ammonia excretion can be in excess of 33 $\mu \mathrm{Eq} / \mathrm{min}$. After studying a number of cases with this method the longer test of calcium chloride loading was preferred. In this test each patient received chloride $150 \mathrm{mEq} /$ day for 3 days, thereafter the urine was tested for urinary $\mathrm{pH}$, titratable acidity and ammonia excretion. All diuretics were stopped at least 5 days before any acid loading test and hypokalaemia was corrected with oral potassium before any test was performed. Urinary amino acids were detected by two dimensional paper chromatography, plasma copper levels were measured on an atomic absorption spectrometer, the normal range being $70-150 \mu \mathrm{g} / 100 \mathrm{ml}$. Autoantibodies were detected by an immunofluorescent technique using a $1: 10$ dilution of the patient's serum. Serum immunoglobulins were measured quantitatively by the single radial immunodiffusion method on an immunoplate using standard supply by the manufacturer (Baxter Thetford).

\section{Results}

The incidence of renal tubular acidosis occurring in the four diagnostic groups comprising the 117 patients studied is shown in Table 2 . The highest incidence of the incomplete type of renal tubular acidosis was found in those with primary biliary cirrhosis and active chronic hepatitis. Similarly the incidence of overt acidosis was also highest in these two groups. Only one patient with alcoholic cirrhosis was found to have an incomplete type of renal tubular acidosis. In all, thirty-eight patients $(32 \%)$ had overt or incomplete tubular defect.

Patients with overt acidosis had symptoms of polydypsia, polyuria, muscular weakness, nausea and vomiting. In two cases these symptoms were responsible for the initial presentation in hospital before liver disease was diagnosed. In the other nine overt cases the tubular defect became apparent during the course of their liver disease. Hypokalaemia with an inappropriate loss of urinary potassium (44-105 $\mathrm{mEq}$ in $24 \mathrm{hr}$ ) occurred in seven patients with overt acidosis, whilst osteomalacia was seen in two cases. Proteinuria occurred in ten patients with either the incomplete or overt forms. The protein loss was not excessive and did not exceed $2 \mathrm{~g}$ in $24 \mathrm{hr}$. Specific tests for tubular protein loss were not performed. Aminoaciduria was not seen in any patient and glycosuria was only noted in three patients with diabetes. Nephrocalcinosis was seen in two patients and in both of these urinary and plasma calciums were normal (Fig. 1).

There was no correlation between the level of globulin in those with and those without the tubular defect (Fig. 2), and likewise there was no difference in the globulin levels between those with overt and those with incomplete acidosis. Similarly there was no significant difference in the serum copper level in those with and those without the defect (Fig. 3).

Qualitative changes in the pattern of acid excretion were noted in many patients with acidifying defects. Titratable acidity was below normal in all cases and the ammonia excretion accounted for an increased percentage of total hydrogen ion excretion. Patients with renal tubular acidosis excreted excessive amounts of ammonia at a given urinary $\mathrm{pH}$ (Fig. 4). However, in those with overt acidosis a normal ammonia excretion for the urinary $\mathrm{pH}$ was found. In one patient, tested on admission to hospital, an incomplete type of distal tubular acidosis was found. She was excreting an excess of ammonia at $120 \mu \mathrm{g} / \mathrm{min}$ at a urine $\mathrm{pH}$ of $5 \cdot 7$. Two months later she developed a metabolic acidosis and the urine $\mathrm{pH}$ was found to be $6 \cdot 2$. At this time her ammonia excretion was only $39 \mathrm{mEq} / \mathrm{min}$. This apparent drop in ammonia excretion had occurred without any significant fall in the glomerular filtration rate. Creatinine clearance was measured in a large percentage of the cases (Fig. 5) and was found to be of the same order in those with and those without renal tubular acidosis, although it tended to be lower in those with the overt variety of the distal tubular defect. It has been suggested that the increased ammonia excretion in patients with renal tubular acidosis is due to an increased creatinine clearance, however, as can be seen from Fig. 5, our findings do not confirm this.

\section{Immunological studies}

The presence of mitochondrial, smooth muscle and antinuclear antibodies, as well as the numbers of

TABLE 2. Renal tubular acidosis and chronic liver disease

\begin{tabular}{lcccc}
\hline & No. & Incomplete RTA & Overt RTA & Total abnormal $(\%)$ \\
\hline Active chronic hepatitis & 48 & 11 & 4 & 31 \\
Primary biliary cirrhosis & 29 & 11 & 6 & 59 \\
Cryptogenic cirrhosis & 25 & 4 & 1 & 20 \\
Alcoholic cirrhosis & 15 & 1 & 0 & 7 \\
\hline
\end{tabular}




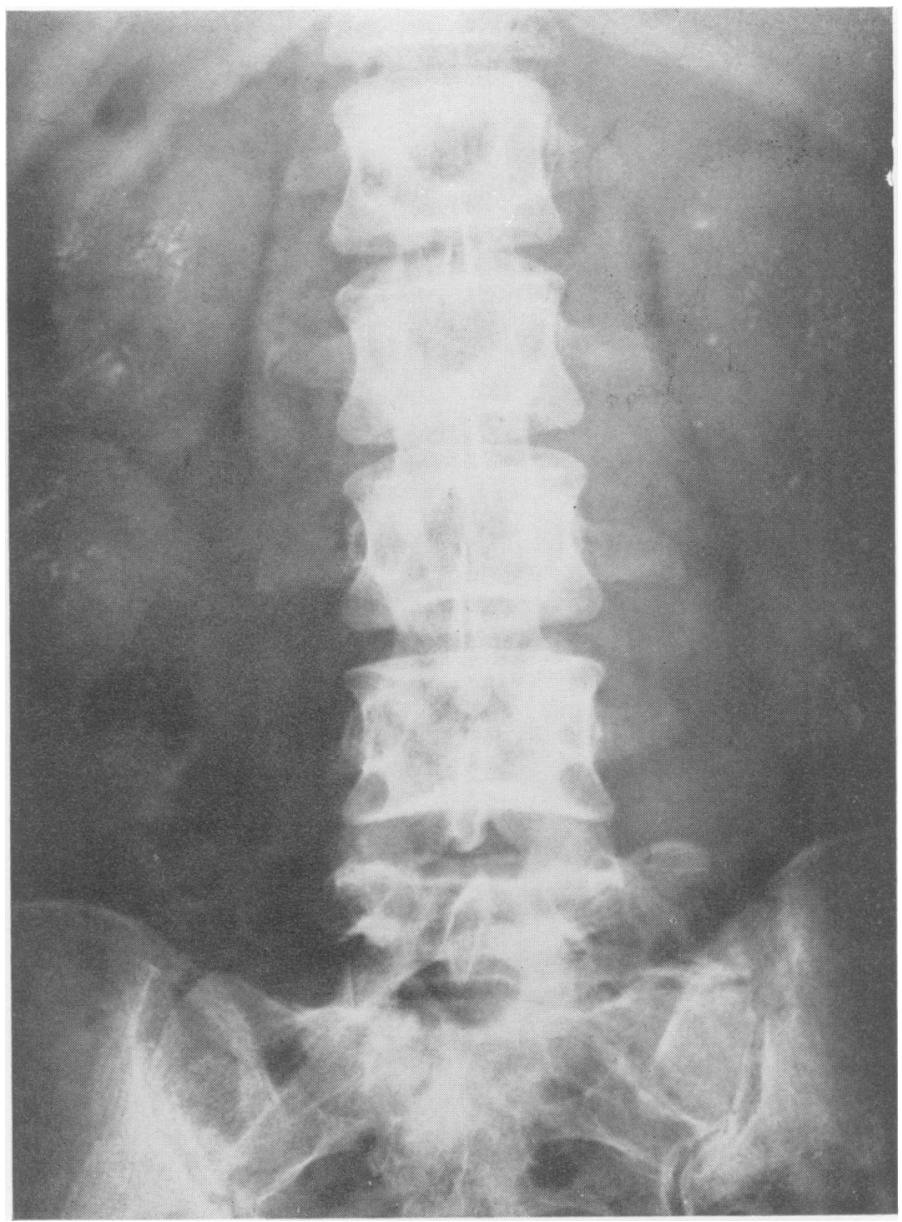

FIG. 1. Straight X-ray of the abdomen in a patient with overt renal tubular acidosis showing nephrocalcinosis.

patients with abnormal levels of $\operatorname{IgA}$, IgG and IgM are shown in Table 3. It can be seen that the presence of renal tubular acidosis could not be significantly associated with abnormal levels, class of immunoglobulin, nor with any particular autoantibody.

Renal histology was obtained in eight patients with renal tubular acidosis and revealed an interstitial nephritis in seven, characterized by collections of lymphocytes with some fibrosis throughout the renal cortex (Fig. 6). In two patients there was also a diffuse calcinosis. Glomerular changes were minimal although in two other patients, one with overt renal tubular acidosis and one with normal renal function, immunofluorescent techniques showed linear staining of the basement membrane due to antibody deposition. This infiltration of the kidneys by lymphocytes could possibly indicate that cellmediated immune mechanisms might be responsible for the tissue damage and the tubular defect. This was investigated using the leucocyte migration inhibition test, details of which are described elsewhere (Mitchell et al., 1972; Smith et al., 1972; Soborg, 1968). Abnormalities of leucocyte migration were confined to those patients with renal tubular acidosis in the active chronic, primary biliary cirrhosis and cryptogenic cirrhotic groups. No abnormality was found in patients with alcoholic cirrhosis. Of the twenty-one patients with renal tubular acidosis tested, $62 \%$ were abnormal to renal antigens, whereas of thirty-four patients without the tubular defect only $6 \%$ showed an abnormal migration. It has previously been shown that patients with these types of liver disease have abnormal leucocyte migration when using liver antigens (Smith et al., 1972) and it therefore could be argued that the inhibition of migration, seen in patients with renal tubular 


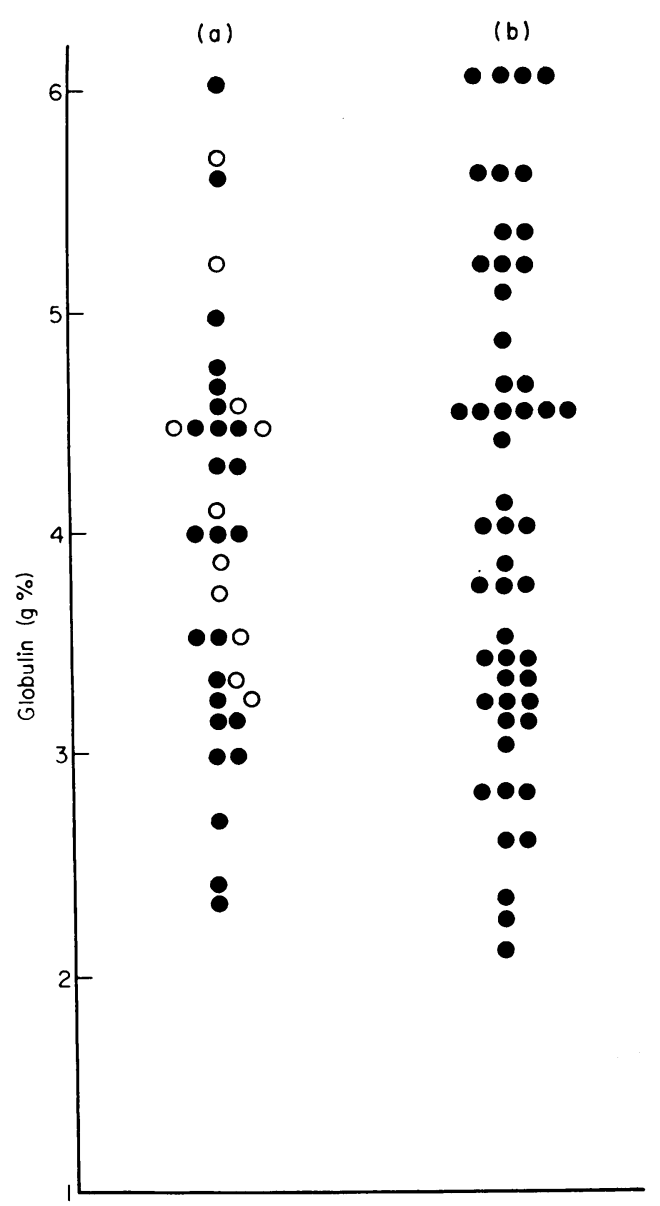

FIG. 2. Serum globulin in chronic liver disease. (a) With RTA; (b) without RTA. $\mathrm{O}=$ overt RTA.

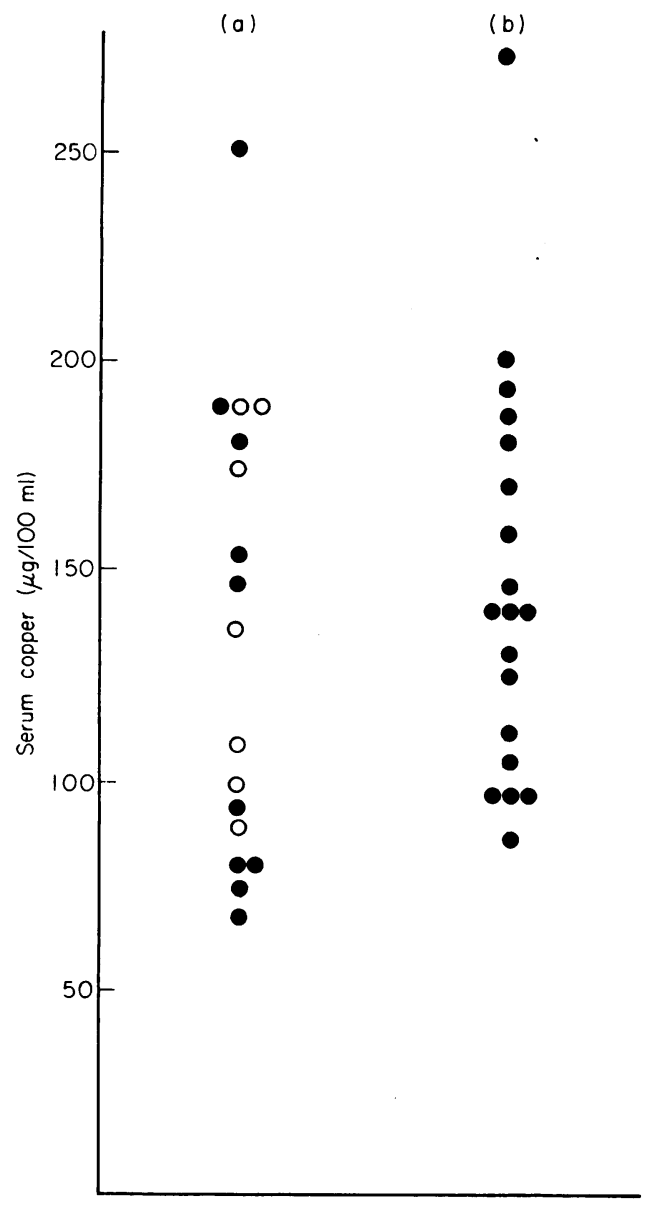

FIG. 3. Serum copper in chronic liver disease. (a) With RTA; (b) without RTA. O = overt RTA.

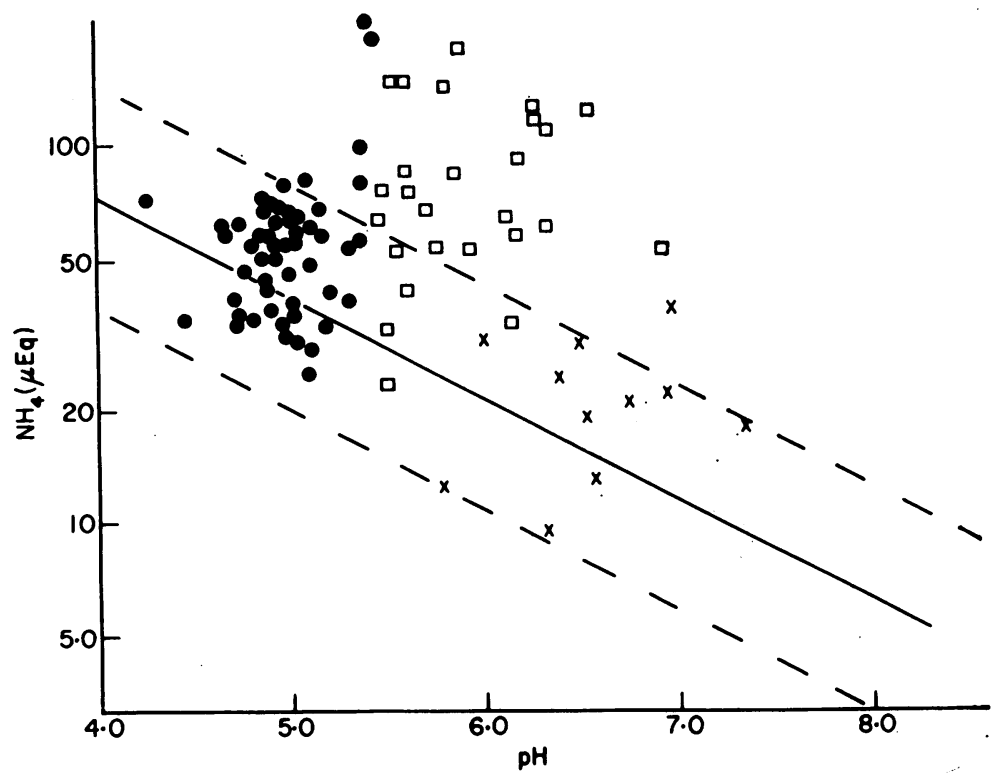

FIG. 4. Ammonia excretion in chronic liver disease. $\square$, incomplete RTA; $\times$, overt RTA; $\bigcirc$, normal tubular function. 


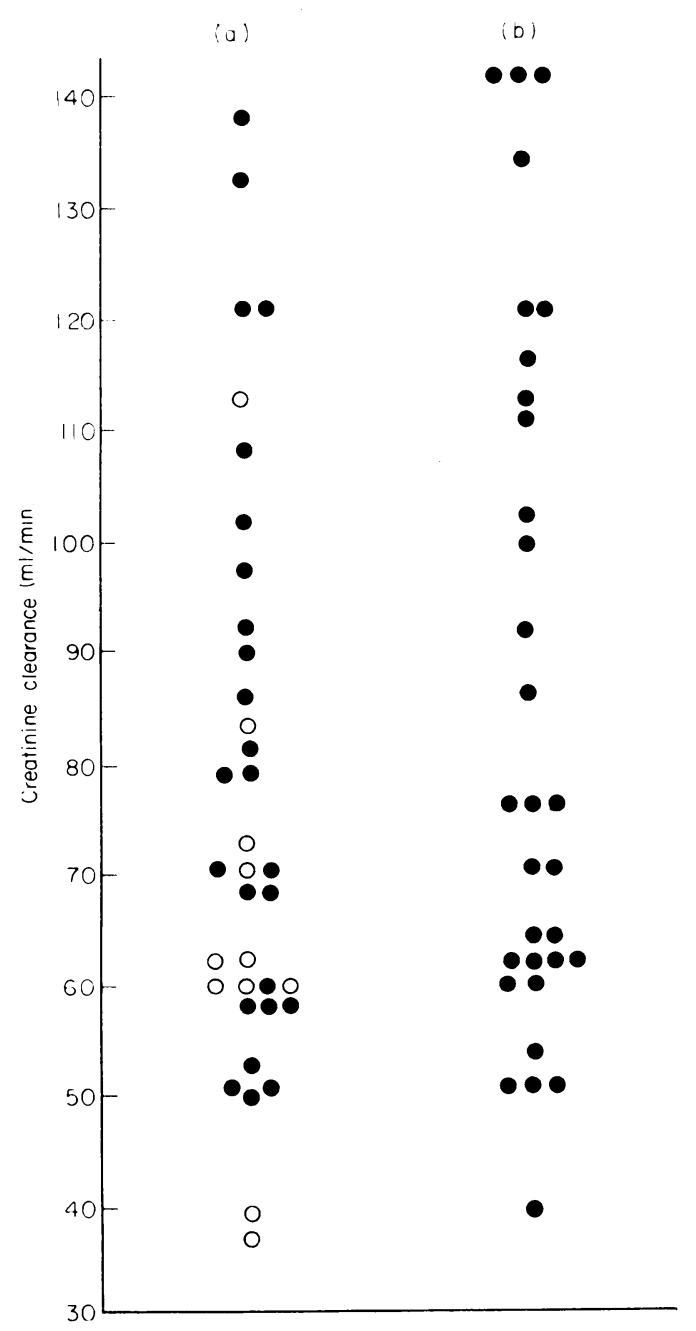

FIG. 5. Creatinine clearance in chronic liver disease. (a) With RTA; (b) without RTA. O = overt RTA.

TABLE 3. Immunoglobulins, autoantibodies and RTA

\begin{tabular}{lcc}
\hline & RTA present & RTA absent \\
\hline IgA (\% abnormal) & 49 & 52 \\
IgG & 45 & 55 \\
IgM & 58 & 40 \\
Mitochondrial antibodies $(\%)$ & 54 & 44 \\
Smooth muscle antibodies $(\%)$ & 30 & 32 \\
Antinuclear factor & 53 & 41 \\
\hline
\end{tabular}

acidosis and autoimmune liver disease, could simply be due to cross-reactivity. However, if one compares the reaction to renal antigens and liver antigens in the same patients (Fig. 7), it can be seen that there are four possible reactions. There are some that are normal to both liver and kidney antigens, some that are abnormal to liver but normal to renal antigens, a third group who are normal to liver but abnormal to renal antigens and, finally, a group abnormal to both antigens. In the latter group all the patients had rena tubular acidosis. This figure shows, therefore, that cross-reactivity cannot be the whole answer to this abnormal leucocyte migration.

\section{Discussion}

A defect of urinary acidification was found in $32 \%$ of patients with chronic liver disease. This defect was of the distal tubular or gradient type and presented either as an incomplete or an overt variety. It would appear that patients with the incomplete type are able to maintain an acid base balance by excreting larger quantities of ammonium ion, but the reason for this is unknown. It cannot be due solely to an abnormal creatinine clearance. Anything which impairs ammonium excretion in these patients is likely, therefore, to impair the excretion of total hydrogen ion thereby inducing an overt metabolic acidosis. The fall in creatinine clearance could explain this in some of the overt cases, as shown in Fig. 5, but not in all. It is possible that the impaired ammonium excretion could reflect greater damage, by some unknown means, to the distal renal tubule.

In potassium deficiency the ability of the kidney to excrete a maximally acid urine is impaired bu ammoniun excretion remains normal (Clark et al 1955). Hypokalaemia was found in seven cases with overt acidosis though all of these had an inappropriate loss of urinary potassium. However renal tubular acidosis itself is a known cause of hypokalaemia (Fourman and McCance, 1955). In the patients with the incomplete variety, hypokalaemia had only occurred at any time in twelve patients and all of these had been corrected with potassium supplements well before urinary testing. Thus, although it is possible that intracellular hypokalaemia may have existed, it could only account for a small number of patients with tubular acidosis. A disturbance of the peritubular circulation due to hyperglobulinaemia has been suggested as a cause of renal tubular acidosis but there was no correlation between the level of the serum globulin and the presence of the disorder thus confirming the findings of Morris and Fudenberg (1967), Shearn and Tu (1968) and Tu et al. (1968). Leeson and Fourman (1967) suggested that in a patient with primary biliary cirrhosis and tubular acidosis a defect of acidification was due to the deposition of copper in the renal tubules. However, it is unlikely that an abnormality of copper metabolism is a major factor in the development of the renal lesion in our patients. Muldowney, Freaney and McGeeney (1968) found renal tubular acidosis in five cases of osteomalacia and suggested that the renal lesion might be due to a deficiency of vitamin $D$ causing secondary hyperparathyroidism rather than 


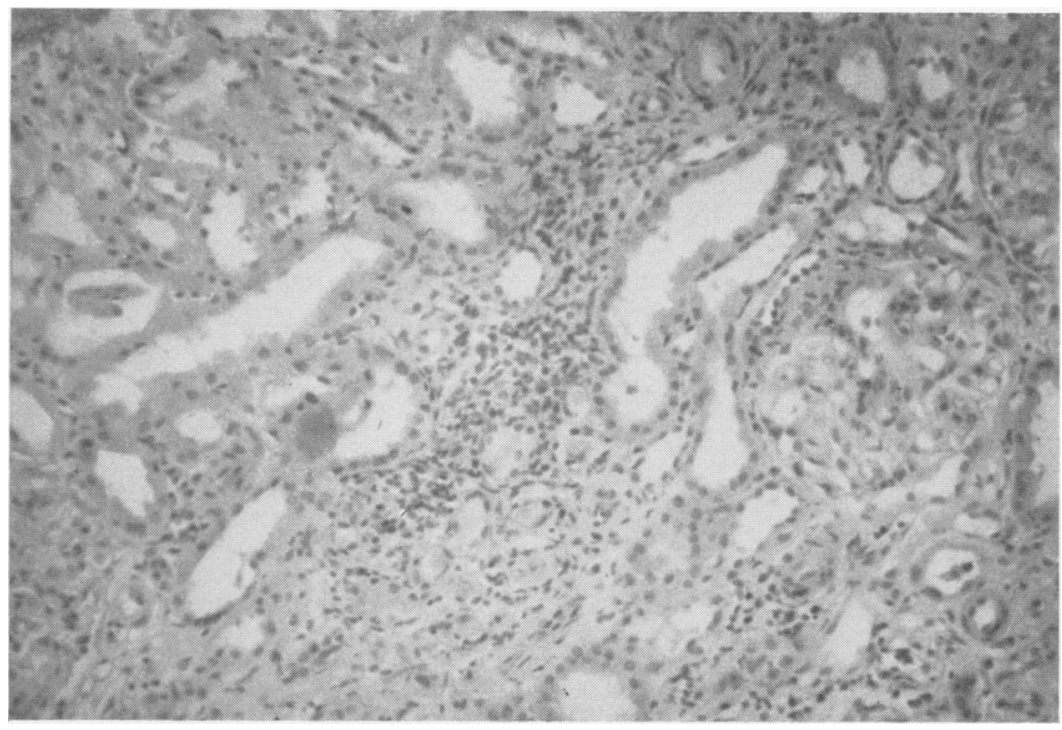

FIG. 6. Renal biopsy of patient with overt renal tubular acidosis showing lymphocytic infiltration and tubular atrophy.

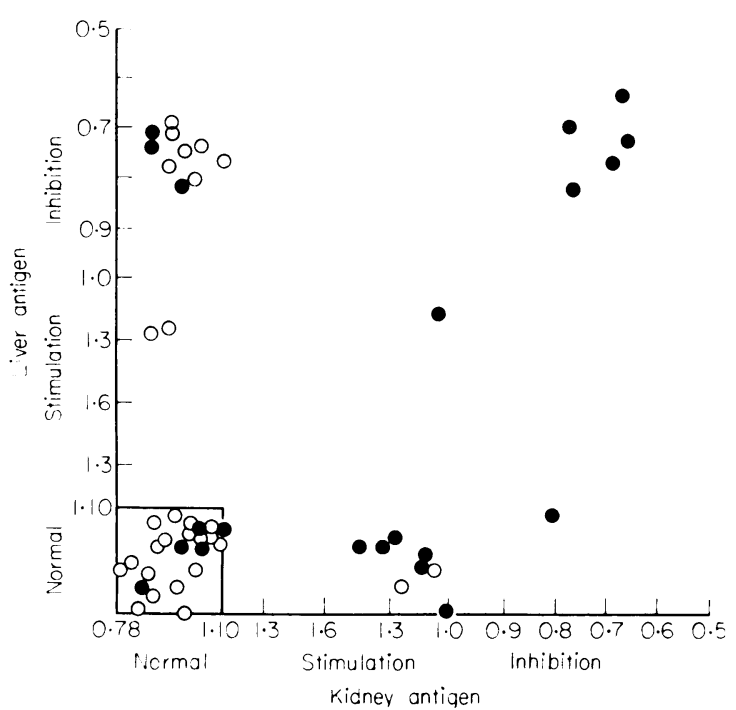

FIG. 7. Comparison of leucocyte migration in the same patients using liver antigen and kidney antigen. 0 , Renal tubular acidosis.

a deficiency of vitamin $\mathrm{D}$ per se. Osteomalacia was only present in two of our cases and in neither of these did the tubular defect improve with administration of vitamin $D$ over a prolonged period; furthermore the tubular lesion in these cases is usually a proximal lesion in which aminoaciduria and glycosuria would be expected. In none of our cases was any abnormal aminoaciduria found.
Increasing evidence points to an immunological lesion as causing at least some cases of renal tubular acidosis (Carter, Whitworth and Mackay, 1971) and in this context an association between the Sjögren's syndrome and renal tubular acidosis is now well recorded (Shioji et al., 1970). In several such patients and in patients with renal tubular acidosis in association with other immunological disease, lymphocytic infiltration of the renal cortex with tubular destruction has been demonstrated (Pasternack and Linder, 1970; Kaltrieder and Talal, 1969; Tu et al., 1968). In the present series, Sjögren's syndrome and renal tubular acidosis occurred together in $27 \%$ of the patients. The result, with renal antigens, suggests that cell-mediated responses are involved in the pathogenesis of the tubular defect. It is perhaps relevant here that renal tubular acidosis has been described in renal transplant patients in whom cellmediated responses were thought to be responsible for rejection episodes (Feldman and Lee, 1967). Furthermore, tubular lesions, in rabbits immunized with renal antigens, are associated with mononuclear cell infiltration of the kidney (Klassen, McCluskey and Milogrom, 1971) similar to that seen in these patients with renal tubular acidosis. Overall, the association between renal tubular acidosis and chronic liver disease could be due to multiple factors. Electrolyte abnormalities in patients with liver disease could explain a tubular defect in some patients but immunological factors probably play a major role. 


\section{Acknowledgments}

I would like to thank Dr J. Bamforth, Dr A. Dawson, Dr R. Williams and the Physicians of the London Hospital for permission to study their cases and also Mr McLean of the Photographic Department, Royal Portsmouth Hospital, for producing the plates and figures.

\section{References}

Carter,N. G., Whitworth, J.A. \& Mackay, I.R. (1971) Impaired urinary acidification, its incidence in diseases with autoimmune features. Australian and New Zealand Journal of Medicine, 1, 39.

Clarke, E., Evans, B.M., Macintyre, I. \& Milne, M.D. (1955) Acidosis in experimental electrolyte depletion. Clinical Science, 14, 421.

Feldman, J.D. \& LeE, S. (1967) Renal homotransplantation in rats. 1. Allogenic recipients. Journal of Experimental Medicine, 126, 783.

Fourman, P. \& MCCance, R.A. (1955) Tetany complicating the treatment of potassium deficiency in renal acidosis. Lancet, i, 329.

Golding, P.L. \& Mason, A.S.M. (1971) Renal tubular acidosis and autoimmune liver disease. Gut, 12, 153.

Kaltrieder, H.B. \& TAlal, N. (1969) Impaired renal acidification in Sjögren's Syndrome and related disorders. Arthritis and Rheumatism, 12, 538.

Klassen, J., MCCluskey, R.T. \& Milogrom, F. (1971) Nonglomerular renal disease produced in rabbits by immunization with homologous kidney. American Journal of Pathology, 63, 333.

LeEson, P.M. \& Fourman, P. (1967) A disorder of copper metabolism treated with penicillamine in a patient with primary biliary cirrhosis and renal tubular acidosis. American Journal of Medicine, 43, 620.

Mitchell, C.G., Smith, M.G.M., Golding, P.L., EddleSTONE, A.L.W.F. \& Williams, R. (1972) Evaluation of leucocyte migration test as a measure of delayed hypersensitivity in man suppression of migration inhibition by puromycin. Clinical and Experimental Immunology, 11, 535.

MORRIS, R.C., JR (1969) Renal tubular acidosis. New England Journal of Medicine, 281, 1405.

MorRIs, R.C. \& FudenberG, H.H. (1967) Impaired renal acidification in patients with hypergammaglobulinaemia. Medicine (Baltimore), 45, 57.

Muldowney, F.P., Freaney, R. \& McGeeney, D. (1968) Renal tubular acidosis and aminoaciduria in osteomalacia of dietary or intestinal origin. Quarterly Journal of Medicine, 37, 517.

Pasternack, A. \& Linder, E. (1970) Renal tubular acidosis, an immunopathological study on four patients. Clinical and Experimental Immunology, 7, 115.

Read, A.E., Sherlock, S. \& Harrison, C.V. (1963) Active juvenile cirrhosis considered as part of a systemic disease and the effect of corticosteroid therapy. Gut, 4, 378.

SeEdat, Y.K. \& RaINE, E.R. (1965) Active chronic hepatitis associated with renal tubular acidosis and successful pregnancy. South African Medical Journal, 39, 595.

Shear, L., BONKOWSKY, H.L. \& Gabuzda, C.J. (1969) Renal tubular acidosis in cirrhosis. New England Journal of Medicine, 280, 1.

SHEARN, M.A. \& TU, W.H. (1968) L.atent renal tubular acidosis in Sjögren's syndrome. Annals of Rheumatic Diseases, 27, 27.

Shioji, R., Furuyama, T., Onodera, S., Saitu, H., Ito, H. \& SASAKI, Y. (1970) Sjögren's Syndrome and renal tubular acidosis. American Journal of Medicine, 48, 456.

Smith, M.G.M., Golding, P.L., EdDleston, A.L.W.F., Mitchell, C.G., Kemp, A. \& Williams, R. (1972) Cell mediated immune responses in chronic liver disease. Britis? Medical Journal, 1, 572.

SOBORG, M. (1968) In vitro migration human peripheral leu cocytes in cellular hypersensitivity. Acta medica scandi navica, 184, 135.

Tu, W.H., Shearn, M.A., Lee, J.C. \& HopPer, J. (1968) Interstitial nephritis in Sjögren's Syndrome. Annals of Internal Medicine, 69, 1163.

WRONG, O.M. \& DAVIES, H.E.F. (1959) The excretion of acid in renal disease. Quarterly Journal of Medicine, 28, 259. 